\title{
Improved Temporal Resolution for Functional Studies with Reduced Number of Segments with Three- Dimensional Echo Planar Imaging
}

\author{
Mayur Narsude, ${ }^{1,3}$ Wietske van der Zwaag, ${ }^{1}$ Tobias Kober, ${ }^{1,2,3}$ Rolf Gruetter, ${ }^{1,3,4}$ and \\ José P. Marques ${ }^{3 *}$
}

Purpose: To introduce a new k-space traversal strategy for segmented three-dimensional echo planar imaging (3D EPI) that encodes two partitions per radiofrequency excitation, effectively reducing the number excitations used to acquire a 3D EPI dataset by half.

Methods: The strategy was evaluated in the context of functional MRI applications for: image quality compared with segmented 3D EPI, temporal signal-to-noise ratio (tSNR) (the ability to detect resting state networks compared with multislice two-dimensional (2D) EPI and segmented 3D EPI, and temporal resolution (the ability to separate cardiac- and respiration-related fluctuations from the desired blood oxygen level-dependent signal of interest).

Results: Whole brain images with a nominal voxel size of 2 $\mathrm{mm}$ isotropic could be acquired with a temporal resolution under half a second using traditional parallel imaging acceleration up to $4 \times$ in the partition-encode direction and using novel data acquisition speed-up of $2 \times$ with a $32-$ channel coil. With $8 \times$ data acquisition speed-up in the partition-encode direction, $3 D$ reduced excitations (RE)-EPI produced acceptable image quality without introduction of noticeable additional artifacts. Due to increased tSNR and better characterization of physiological fluctuations, the new strategy allowed detection of more resting state networks compared with multislice 2D-EPI and segmented 3D EPI.

Conclusion: 3D RE-EPI resulted in significant increases in temporal resolution for whole brain acquisitions and in improved physiological noise characterization compared with 2D-EPI and segmented 3D EPI. Magn Reson Med 72:786-792, 2014. (c) 2013 Wiley Periodicals, Inc.

Key words: k-space trajectory; pulse sequence; functional MRI; temporal SNR; physiological noise; acceleration; EPI

\footnotetext{
${ }^{1}$ Laboratory for Functional and Metabolic Imaging, Ecole Polytechnique Fédérale de Lausanne, Lausanne, Switzerland.

${ }^{2}$ Advanced Clinical Imaging Technology, Siemens Medical Solutions-CIBM, Lausanne, Switzerland.

${ }^{3}$ Department of Radiology, University of Lausanne, Lausanne, Switzerland. ${ }^{4}$ Department of Radiology, University of Geneva, Geneva, Switzerland. Grant sponsors: Centre d'Imagerie BioMédicale of the University of Lausanne; Grant sponsor: Swiss Federal Institute of Technology Lausanne; Grant sponsor: University of Geneva; Grant sponsor: Centre Hospitalier Universitaire Vaudois; Grant sponsor: Hôpitaux Universitaires de Genève; Grant sponsor: The Leenaards and Jeantet Foundations.

*Correspondence to: José P. Marques, Station 6, CH-1015 Lausanne, Switzerland. E-mail: jose.marques@epfl.ch

Received 14 May 2013; revised 23 August 2013; accepted 6 September 2013

DOI 10.1002/mrm.24975

Published online 17 October 2013 in Wiley Online Library (wileyonlinelibrary. com).
}

(c) 2013 Wiley Periodicals, Inc.

\section{INTRODUCTION}

Echo planar imaging (EPI) (1) is the main pulse sequence used for functional MRI (fMRI) $(2,3)$. At high magnetic field strengths $(\geq 7 \mathrm{~T})$, due to the increased signal available and because of the increased susceptibility-induced artifacts, there is both an opportunity and a need to acquire higher spatial resolution. Increasing spatial resolution of multislice two-dimensional (2D) EPI implies an increase not only in the echo train length (ETL) but also in the number of slices needed to achieve the same volume coverage, thereby increasing the minimum pulse repetition time (TR) achievable for whole brain coverage to a point where it becomes comparable to the hemodynamic response.

Echo volume imaging or single-shot three-dimensional (3D) EPI $(4,5)$ is a technique where a thick slab is excited and multiple partitions are encoded within the thick slab during a single readout. 3D single-shot acquisitions require long ETL, which compromises the echo time (TE) and spatial resolution achievable, or have high demands on the gradient hardware (6). One strategy that has been proposed for reducing ETL is to perform multislab echo volume imaging (7). Dividing the volume in the partition-encode direction into $\mathrm{N}$ slabs reduces ETL by a factor of N; however, slices at the edge of each slab often have to be discarded because of a poor slice profile, which reduces the ability to use parallel imaging in the slab-encoding direction due to the reduced field of view. Alternate to conventional Cartesian trajectories, single-shot 3D k-space coverage has been proposed using either concentric shells trajectory (8) or 3D Rosette trajectory (9). These new trajectories imply computationally intensive iterative reconstruction schemes.

A more established strategy to overcome the limitations of echo volume imaging is the acquisition of the 3D volume in several shots (10-12), known as segmented 3D EPI. In this case, time needed to acquire one entire 3D $\mathrm{k}$-space $\left(\mathrm{TR}_{\text {volume }}\right)$ is $\mathrm{TR}_{\text {segment }}$ times the number of segments, where $\mathrm{TR}_{\text {segment }}$ is the time between successive excitations of the tissue volume. Typically, one single $\mathrm{k}$-space plane is acquired per excitation, leading to ETL comparable to those of 2D-EPI allowing acquisitions with $\mathrm{TE} \sim \mathrm{T}_{2}{ }^{*}$. Despite its reduced steady state magnetization due to the reduced $\mathrm{TR}_{\text {segment }}$ and Ernst angle, segmented 3D EPI (12) provides multiple benefits over multislice 2D-EPI approach such as a higher sensitivity per unit scan time $(13,14)$, the absence of a spin-history artifact (11), and the possibility of applying parallel imaging technique in both phase-encode directions (12). On the other hand, the maximum available temporal 
signal-to-noise ratio (tSNR) is reduced compared with single-shot acquisition $(12,13,15)$ due to physiological processes (15). This loss in tSNR can be recovered using physiological noise correction techniques $(16,17)$, and a tSNR superior to 2D-EPI can be achieved (18).

Recently, multiband excitations have been proposed to accelerate the acquisition of 2D multislice imaging (19) and EPI datasets (20-22) in which simultaneously acquired slices can be separated using coil sensitivity profiles (22). Multiplexed-EPI (23) combines multislice excitation with simultaneous echo refocused EPI (24) at the cost of an increased ETL. These recent approaches have shown their potential to provide subsecond temporal resolution.

The aim of the present study was to develop an acquisition scheme, referred to here as 3D reduced excitations (RE)-EPI, which encodes two k-space planes per radiofrequency (RF) excitation (segment), effectively reducing the number of segments used to acquire the same 3D dataset by half while increasing ETL by a factor of 2 compared with 3D EPI. Image quality assessment was performed for 3D RE-EPI in combination with partial parallel acquisition (PPA) and partial Fourier (PF) with different overall data acquisition speed-up factors. tSNR was assessed using resting state network (RSN) detection for 3D RE-EPI and compared with segmented 3D EPI and multislice 2D-EPI acquisitions. The ability to better characterize physiological signal with 3D RE-EPI was demonstrated through power spectral analysis of physiological data and MR signal.

\section{THEORY}

The proposed sequence 3D RE-EPI (Fig. 1b) is a modified version of the segmented 3D EPI sequence (Fig. 1a). In 3D RE-EPI, phase-encode blips are played out after every two readout gradients during the EPI echo train. Partition-encode blips with alternating polarity are played out to encode two successive partitions in a single EPI echo train. Such a k-space trajectory reduces the number of excitations and time needed to cover the imaging volume. The acquisition speed-up factor with $3 \mathrm{D}$ RE-EPI $\left(\mathrm{S}_{\mathrm{PU}}\right)$ can be combined with conventional acceleration methods such as partial Fourier $\left(A_{P F}\right)$ and parallel imaging $\left(A_{P P A}\right)$. The speed-up factor (S) due to this novel acquisition technique, which provides a way to acquire $3 \mathrm{D}$ dataset faster at the cost of increased ETL, must not be confused with parallel imaging acceleration factors (A), which generally result in a reduction in ETL.

\section{METHODS}

All experiments were conducted according to procedures approved by the institutional review board and all participants provided written informed consent prior to experiments. Six healthy subjects (three males and three females; average age, $25.8 \mathrm{yr}$ ) were scanned on a 7T/680 mm head-only scanner (Magnetom, Siemens, Germany) equipped with a head gradient insert (maximum gradient strength and slew rate: $80 \mathrm{mT} / \mathrm{m}, 333 \mathrm{~T} / \mathrm{m} / \mathrm{s})$. A 32-

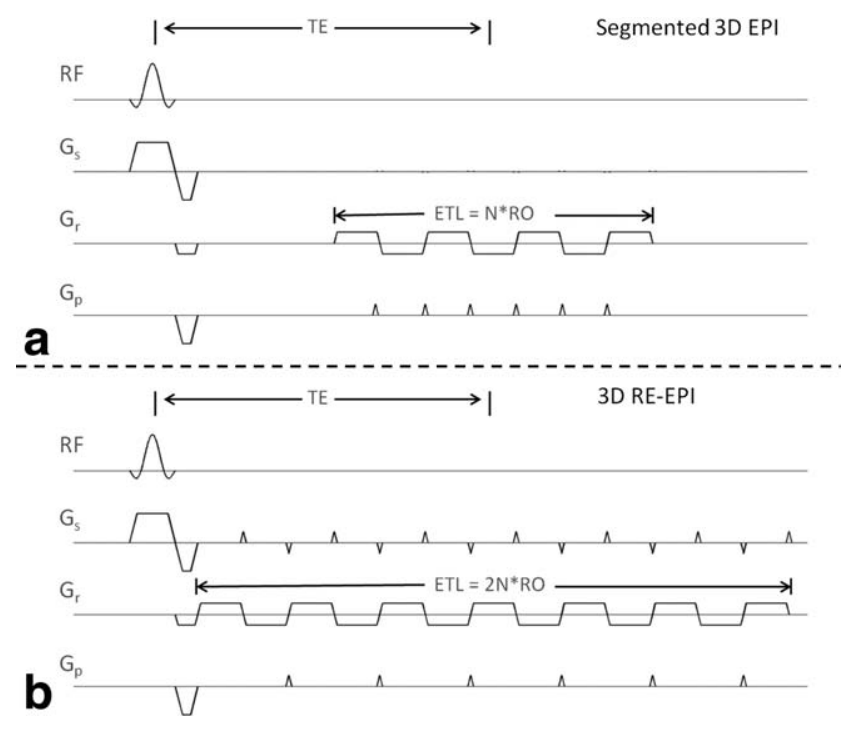

FIG. 1. Pulse sequence diagrams for segmented 3D EPI (a) and 3D RE-EPI sequence (b). In 3D RE-EPI, the phase-encode blips are played out after every two readout blips and the partitionencode blips are played out with alternating polarity after every readout blip so that two adjacent partitions are encoded in a single RF excitation. The ETL becomes double that of the segmented 3D EPI. When the TE is kept constant, the minimum TR for 3D $\mathrm{RE}-\mathrm{EPI}$ becomes $25 \%$ longer compared with segmented 3D EPI.

channel RF-head coil (Nova Medical Inc., Wilmington, Massachusetts, USA) was used for RF reception.

Image quality was evaluated in whole brain images acquired with 3D RE-EPI with $1.2 \mathrm{~mm}$ isotropic nominal resolution (using $156 \times 156 \times 120$ acquisition matrices for the fully sampled data). The overall 3D RE-EPI speed-up $\left(S_{\text {ov }}\right)$ in the partition-encode direction was varied from 1 to 8 using different combinations of $S_{\mathrm{PU}}, \mathrm{A}_{\mathrm{PF}}$, and $A_{\mathrm{PPA}}\left(\mathrm{S}_{\mathrm{OV}}=\mathrm{S}_{\mathrm{PU}} \times \mathrm{A}_{\mathrm{PF}} \times \mathrm{A}_{\mathrm{PPA}}\right)$. Other acquisition parameters were as follows: $\mathrm{TR} / \mathrm{TE}=200 / 30 \mathrm{~ms}$; flip angle $(\mathrm{FA})=17^{\circ}$; readout bandwidth $(\mathrm{rBW})=2190 \mathrm{~Hz} /$ pixel; matrix size $=156 \times 156 \times 120 ; \mathrm{PPA}_{\text {phase }}=3$; and $\mathrm{PF}_{\text {phase }}=6 / 8$ in the phase-encode direction. Frequencyselective fat saturation pulses were applied before each excitation pulse. The partition-encode direction was set to superior-inferior, whereas the phase-encode direction was set anterior-posterior. Image reconstruction was performed entirely online through the vendor-provided software.

An additional low resolution was performed on one of the subjects to assess the effect of fat saturation on the chemical shift artifact, typically observed in echo planar images due to the inherent low bandwidth in the phaseencode direction. The protocol parameters were as follows: sagittal orientation, TR/TE $=40 / 14 \mathrm{~ms} ; \mathrm{FA}=10^{\circ}$; $\mathrm{rBW}=2604 \mathrm{~Hz} /$ pixel; matrix size $=80 \times 80 \times 88$; nominal resolution $=2.5 \mathrm{~mm}$ isotropic; $\mathrm{PPA}_{\text {phase }}=3, \mathrm{PF}_{\text {phase }}$ $=6 / 8$, phase-encode $=$ anterior-posterior. Images were obtained with and without a preparatory spectrally selective fat saturation pulse in two separate runs.

The benefit of improved tSNR with the 3D RE-EPI sequence, compared with multislice 2D EPI and segmented 3D EPI sequences, was evaluated through the detection of RSNs. Common protocol parameters for all 
three sequences: $\mathrm{TE}=26 \mathrm{~ms}, \mathrm{rBW}=2358 \mathrm{~Hz} /$ pixel, matrix size $=106 \times 106 \times 60$, nominal $2 \mathrm{~mm}$ isometric voxels, $\mathrm{PF}_{\text {phase }}=6 / 8, \mathrm{PPA}_{\text {phase }}=2$. Total scan time per sequence was kept constant at 5 min across all the scans. Scans were performed for each subject in a randomized order. Sequence-specific parameters were:

1. Multislice 2D EPI: $\mathrm{TR}_{\text {volume }}=2.920 \mathrm{~s} ; \mathrm{FA}=60^{\circ}$; number of volumes $=102$. The TR used was the minimum possible when sampling the same number of slices at the same resolution as with the segmented 3D EPI.

2. Segmented 3D EPI: $\mathrm{TR}_{\text {segment }}=51 \mathrm{~ms} ; \mathrm{TR}_{\mathrm{volume}}=$ $0.816 \mathrm{~s} ; \mathrm{PF}_{\text {slice }}=6 / 8 ; \mathrm{PPA}_{\text {slice }}=3 ; \mathrm{FA}=13^{\circ}$; number of volumes $=392$.

3. 3D RE-EPI: $\mathrm{TR}_{\text {segment }}=60 \mathrm{~ms} ; \mathrm{TR}_{\text {volume }}=0.480 \mathrm{~s}$; $\mathrm{PF}_{\text {slice }}=6 / 8 ; \mathrm{PPA}_{\text {slice }}=3 ; \mathrm{FA}=14^{\circ}$; number of volumes $=667$.

Anatomical images were acquired with the MP2RAGE sequence (25) using the following parameters: TR $\mathrm{MP}_{\text {2RAGE }}$ $=6.0 \mathrm{~s}$; $\mathrm{TE}=3.03 \mathrm{~ms}$; inversion time $(\mathrm{TI})_{1} / \mathrm{TI}_{2}=0.8 / 2.7 \mathrm{~s}$; $\alpha_{1} / \alpha_{2}=7^{\circ} / 5^{\circ}$; matrix size $=320 \times 320 \times 256$ (nominal 0.6 $\times 0.6 \times 0.6 \mathrm{~mm}^{3}$ isotropic voxels); partition-encode $=$ leftright; phase-encode $=$ anterior-posterior; $\mathrm{PPA}_{\text {phase }}=3$; $\mathrm{PF}_{\text {phase }}=6 / 8 ; \mathrm{PF}_{\text {slice }}=6 / 8$. The Sa2RAGE sequence was used for B1 calibration (26).

fMRI data were motion-corrected using the linear image registration tool FLIRT (FMRIB, Oxford, UK). Datasets were first aligned to their high-resolution anatomical images and subsequently to the MNI152 standard space. RSNs were identified via independent component analysis (ICA) using the MELODIC toolbox (FMRIB) $(27,28)$. Spatial smoothing of 3-mm full-width-half-maximum was applied prior to ICA. Group analysis was performed with multisession temporal concatenation. RSNs that consistently appeared in 2D EPI, 3D EPI, and 3D RE-EPI were automatically identified by choosing the components of the 3D EPI and 3D RE-EPI with spatial correlation $>0.35$ with the 2D EPI RSNs (after thresholding the component maps at $Z=3$ ). When one or more of the 3D EPI and 3D RE-EPI components passed this criterion, the network was dubbed common or split, respectively. Additional independent components were found after visual inspection. RSNs were named via visual comparison with published data (29-32).

tSNR per unit scan time in the white matter and gray matter regions were calculated for all subjects for each run using MATLAB (Mathworks, Natick, Massachusetts, USA). Regions of interest containing $\sim 100$ pixels each were defined in the corpus callosum and cerebral cortex of the sensory motor regions for each subject, and obtained tSNR per unit time values were averaged across subjects. Respiratory and cardiac data were acquired along with MR data from one of the subjects using 3D RE-EPI with $\mathrm{TR}_{\mathrm{volume}}$ of $0.528 \mathrm{~s}$ and using segmented $3 \mathrm{D}$ EPI with $\mathrm{TR}_{\text {volume }}$ of $0.792 \mathrm{~s}$ in two additional runs. Minimum TRs for 3D RE-EPI and segmented 3D EPI were used while keeping TE $\sim \mathrm{T}_{2}{ }^{*}$ (33); this corresponds to a penalty on the $\mathrm{TR}_{\text {segment }}$ by only $\sim 1 / 2$ of the ETL for $3 \mathrm{D}$ RE-EPI when compared with 3D EPI. Power spectrum analysis was performed using MATLAB on both physio- logical data and MR signals to evaluate the benefits of sampling at a higher temporal resolution for physiological noise characterization.

\section{RESULTS}

To determine the overall quality of images, 3D RE-EPI acquisitions were performed with three volunteers and compared with images acquired with segmented 3D EPI. Images in three orthogonal planes (through the center of the acquisition volume of a representative dataset) obtained with different acquisition speed-up factors are shown in Figure 2. Overall acquisition speed-up in the partition-encode direction was calculated as $\mathrm{S}_{\mathrm{Ov}}=\mathrm{S}_{\mathrm{PU}} \times$ $A_{\text {PPA }} \times A_{\text {PF }}$. Nyquist ghosts were visible in the phaseencode direction (anterior-posterior) for segmented 3D EPI and in the partition-encode direction (superior-inferior) with 3D RE-EPI. This resulted from the alternating polarity of the readouts shifting from the phase-encode direction (3D EPI) to the partition-encode direction (3D RE-EPI). Due to the increased ETL, increased distortions in the phase-encode direction (anterior-posterior) were observed.

Figure 3 shows coronal slices at three different positions without (Fig. 3a) and with (Fig. 3b) a preparatory spectrally selective fat saturation pulse. The chemical shift artifacts appeared not only shifted in the phaseencode direction but also as a Nyquist ghost (shifted by $\mathrm{FOV} / 2$ ) in the partition-encode direction, when $3 \mathrm{D}$ RE-EPI was used without fat saturation.

The reduction in $\mathrm{TR}_{\mathrm{volume}}$ resulted in an increase in the number of time points acquired with 3D RE-EPI (667 time points) compared with multislice 2D-EPI (102 time points) and segmented 3D EPI (392 time points), improving the ability to detect RSNs via ICA. The networks detected were divided into three categories: (1) common networks, which included medial visual (red-yellow), executive (blue-light blue), default mode (copper), ventral stream (red), sensory motor (green), left lateral (yellow), and right lateral (pink) (Fig. 4a-4c); (2) split networks, which were found split across multiple independent components with increasing temporal resolution (Fig. 4d-4f); and (3) appearing networks, which were found only at a particular temporal resolution and were absent at the other two temporal resolutions (Fig. 4 g and 4h).

In addition to increased time points, averaged tSNR per unit scan time values were found to be higher, as expected, in 3D RE-EPI $(24.28 \pm 3.42 / 53.81 \pm 3.89$ in white matter/gray matter) compared with 3D EPI (17.48 $\pm 2.14 / 43.78 \pm 3.04$ in WM/GM), an average increase of $\sim 31 \%$ and, compared with multislice 2D-EPI (14.71 \pm $1.32 / 32.22 \pm 0.87$ in $\mathrm{WM} / \mathrm{GM}$ ), an average increase of $\sim 66 \%$. The power spectra of the physiological signals along with the power spectra for the whole brain averaged fMRI time course are shown in Figure 5. Both 1.26 $\mathrm{Hz}$ (segmented 3D EPI, Fig. 5a) and $1.89 \mathrm{~Hz}$ (3D RE-EPI, Fig. 5b) sampling rates were sufficient to sample the first harmonic of respiratory signal $(\sim 0.23 \mathrm{~Hz})$ without aliasing. The first harmonic of the cardiac signal $(\sim 1.20 \mathrm{~Hz})$ was aliased at both the sampling rates used. With the sampling rate of $1.89 \mathrm{~Hz}$, the maximum frequency that 


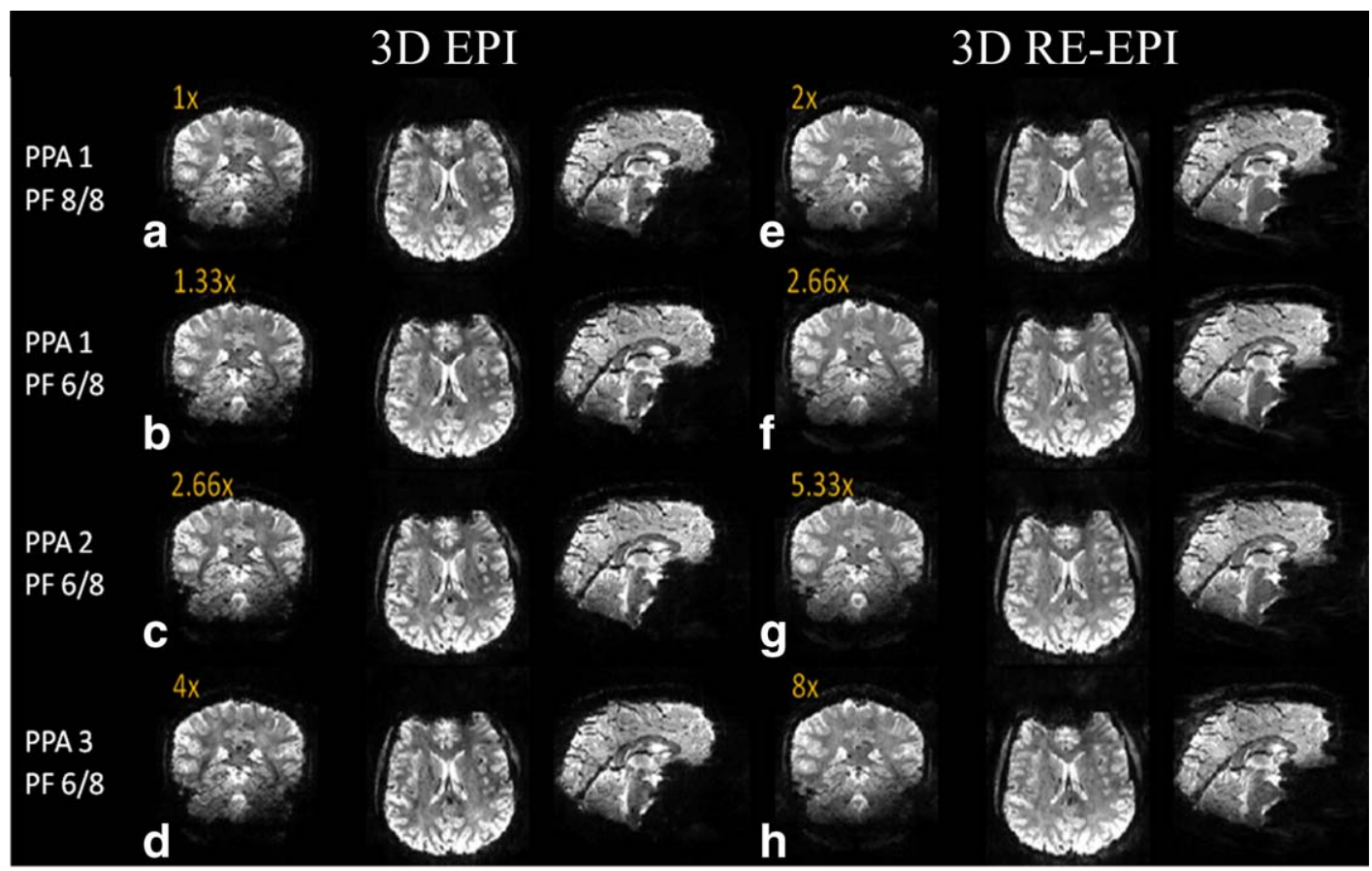

FIG. 2. Each subpanel shows three orthogonal planes from images obtained using 3D EPI and 3D RE-EPI with different overall slicedirection acquisition speed-up factors ranging from $1 \times$ to $8 \times$. The in-plane $A_{P F}$ and $A_{P P A}$ were $6 / 8$ and $3 \times$, respectively, for all images. Overall slice-direction speed-up factors used for each acquisition are indicated in the top left corner of each subpanel (a-h). PPA and $\mathrm{PF}$ acceleration factors used in the partition direction are indicated on the far left. All the data shown were acquired axially with the readout gradient applied along a left-right orientation.

could be sampled without aliasing was $\sim 0.94 \mathrm{~Hz}$, making the aliased peak of the cardiac signal's first harmonic appear at $0.68 \mathrm{~Hz}$ (Fig. 5b) which was away from the blood oxygen level-dependent (BOLD) frequency range (0.04-0.13 Hz), whereas for the 3D EPI, the aliasing occurs at $0.06 \mathrm{~Hz}$ (Fig. 5a), overlapping with the BOLD signal. The insets in Figure 5 show the thresholded power spectra images. The cardiac power was greater in the cerebrospinal fluid and in the proximity of large arterial vessels. In contrast, respiratory signals were observed throughout the brain, since respiration induced $\mathrm{z}$ gradient variations could be observed over the entire brain region (34).

\section{DISCUSSION}

3D RE-EPI provides a speed-up factor of 2 in the partition-encode direction when two partitions per excitation are encoded at an expense of increased echo train length. It should be possible to encode more than two
FIG. 3. Chemical shift artifact with 3D RE-EPI without (a) and with (b) spectrally selective fat saturation preparatory pulse played out before each RF excitation. Chemical shift artifacts (arrows) appear not only shifted in the phase-encode direction but also as Nyquist ghost (shifted by FOV/2) in the partition-encode direction, which was left-right in these sagitally acquired data.

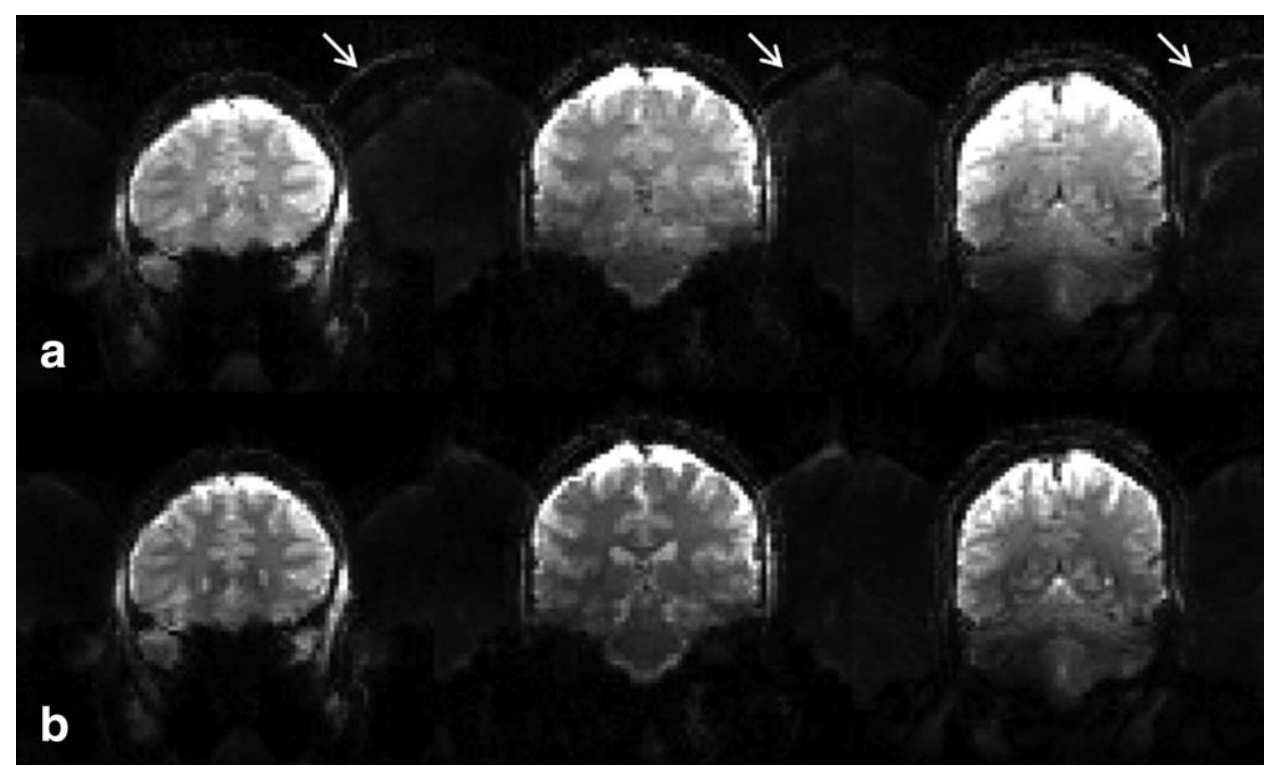


partitions per excitation if stronger gradients or higher phase-encode acceleration is employed. The total reduction of excitations achieved with 3D RE-EPI, in respect to what can be obtained with segmented 3D EPI, is
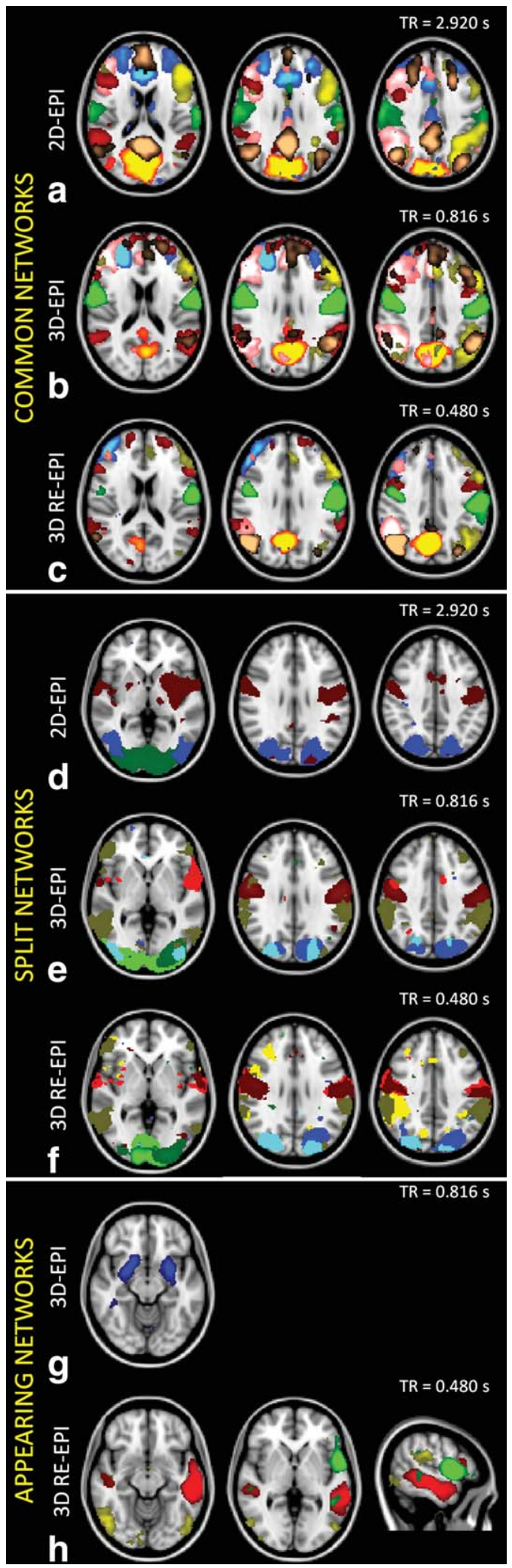

FIG. 4. related with the freedom in distributing the parallelimaging load between the through-plane (partition) and in-plane (phase encoding) directions. Even though 3D RE-EPI doubles ETL $\left(S_{P U}=2\right)$ compared with segmented 3D EPI, thereby increasing the minimum achievable TR, acquisition speed-up is still achieved for fMRI studies. This is because the minimum TR is also limited by TE, which should be kept at $\sim \mathrm{T}_{2}{ }^{*}$ for optimum BOLD signal sensitivity (35), resulting in the minimum TR increment by ETL/2 with 3D RE-EPI. Due to increased ETL, increased distortions are observed in the phase-encode direction. However, these distortions may be reduced by using the same techniques that are developed for 2D-EPI (36-38).

Specific absorption rate (SAR) is one of the limiting factors when imaging at ultra-high field strengths. When accelerating 2D EPI by using multiband excitations, the SAR increases linearly with the number of excited bands, which can limit the maximum SNR achievable. One alternative is to use power independent of number of slices (PINS) excitation pulses $(29,39)$ where the SAR is independent of the number of slices acquired, but the number of slices excited is limited only by the physical dimension of the object and the coverage of the gradient and RF transmit coil along the slice-encode direction. Here, due to the reduced Ernst flip angles and quadratic dependence of SAR on them, the SAR deposition was reduced $\sim 20$-fold when compared with multislice 2D-EPI.

In addition to the higher temporal resolution, the new kspace sampling strategy results in a reduced number of excitations used to form the same $3 \mathrm{D}$ dataset. This is advantageous because it has been shown that the maximum available temporal SNR with segmented 3D EPI decreases with increasing number of segments used (15). Compared with the segmented 3D EPI, 3D RE-EPI results in a significant increase in tSNR, which explains the improvement in the detection of the RSNs shown in Figure 4.

To detect activation-induced BOLD responses, reducing the influence of signal fluctuations caused by cardiac pulsation and respiration is especially important at high SNR. The use of short TRs, as possible with 3D RE-EPI, enables better characterization of these nuisance sources

FIG. 4. Resting state networks shown overlaid on the MNI template. Resting state networks were categorized as common networks (a: 2D-EPI; b: 3D EPI; c: 3D RE-EPI), split networks (d: 2DEPI; e: 3D EPI; f: 3D RE-EPI), or appearing networks (g: 3D EPI; h: 3D RE-EPI). a-c: Common networks, which were seen in all three acquisitions (medial visual [red-yellow], executive [blue-light blue], default mode [copper], ventral stream [red], sensory-motor [green], left lateral [yellow], and right lateral [pink]) are shown on axial slices at Montreal Neurological Institute (MNI) coordinates z $=18,28$, and 38. $\mathbf{d}-\mathbf{f}$ : Split networks, which appear as multiple components in 3D EPI and/or 3D RE-EPI (lateral visual [dark green, green, and light green], auditory [dark red and light red], precuneus [dark blue and light blue], and task-positive network [brown and light yellow]) are shown on shown on axial slices at MNI coordinates $z=0,28$, and 38. g: Appearing network only found in 3D EPI (medial temporal network [blue]) shown at MNI coordinate $z=-12$. $\mathbf{h}$ : Appearing networks only found in 3D REEPI (language [green], MT-centred visual [yellow], and a bilateral superior temporal sulcus, or secondary auditory network [red]) is shown at MNI coordinates $\mathrm{z}=-12,0$ and $\mathrm{x}=-54$. 


\section{Power Spectrum}

FIG. 5. Power spectra of two scans acquired with $3 \mathrm{D}$ RE-EPI at a sampling rate of $1.89 \mathrm{~Hz}$ (a) and segmented 3D EPI at a sampling rate of $1.26 \mathrm{~Hz}$ (b). Separately acquired power spectra of respiratory activity (blue) and cardiac activity (red) are shown for both experiments. Note that BOLD signals are typically found in the $0.04-0.13 \mathrm{~Hz}$ frequency range. Inset: Overlay of pixels contributing signal variations at cardiac rate (upper row) and at respiratory rate (lower row) on three different representative slices from an anatomical dataset. A thresholded power spectrum with a spectral window of $0.66-0.70 \mathrm{~Hz}$ was used to create cardiac pixel overlay, whereas respiratory pixel overlay was created using a spectral window of $0.21-0.25 \mathrm{~Hz}$. [Color figure can be viewed in the online issue, which is available at wileyonlinelibrary.com.]

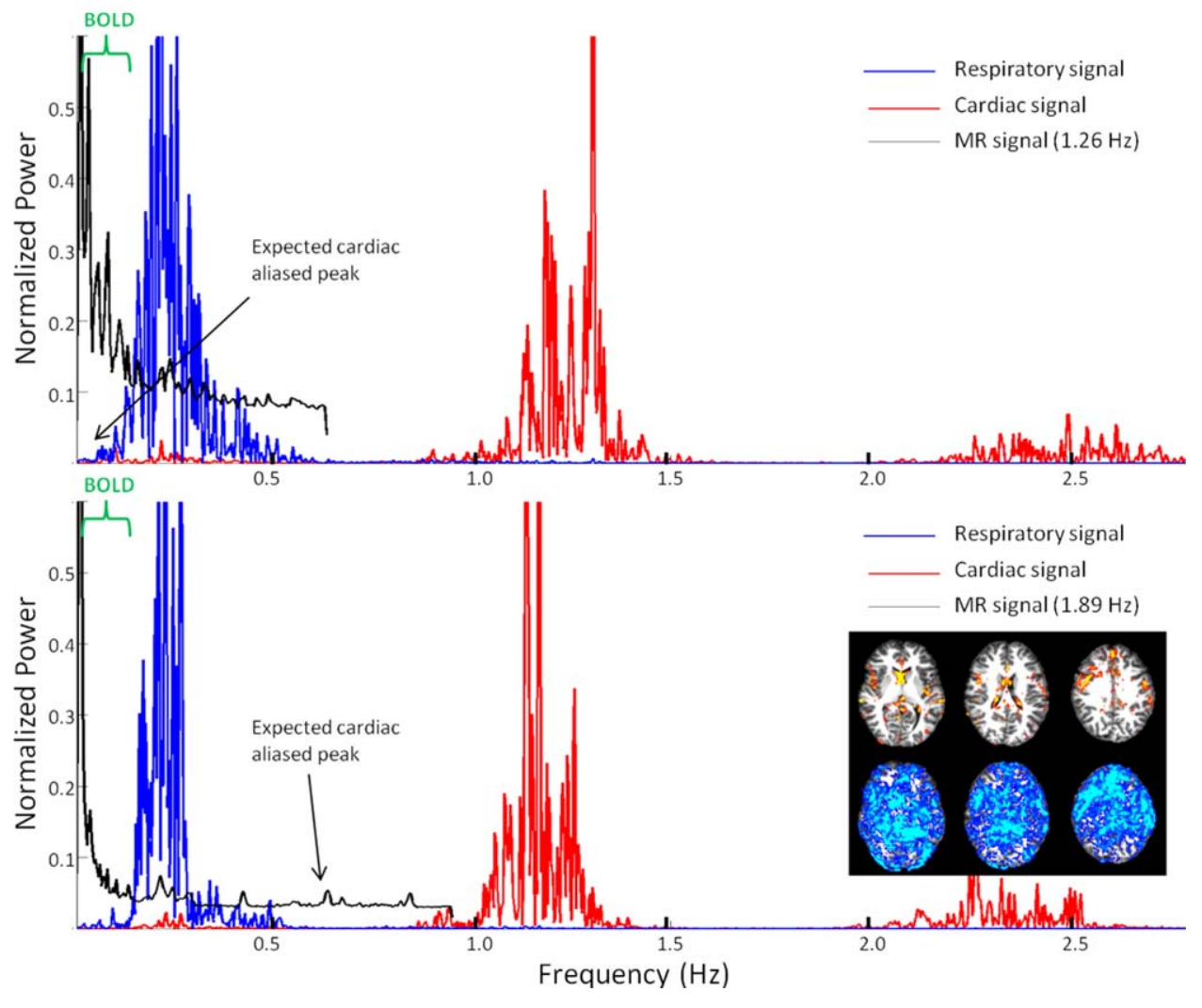

that could then be removed: either by simple low-pass filtering or via the physiological noise reduction schemes $(16,17,40)$. The improvement in detection of RSNs with faster imaging by means of increased temporal resolution and tSNR was demonstrated in the multiplexed 2D EPI (23) at $3 \mathrm{~T}$. The results with $3 \mathrm{D}$ RE-EPI are consistent with those and are the first independent demonstrations at $7 \mathrm{~T}$ that, with 3D imaging, the same principle holds true.

3D RE-EPI is expected to be of special benefit for fMRI applications where temporal resolution, more than spatial resolution, is a limiting factor [e.g., when studying rapid transient responses (41) or the shape of the hemodynamic response function (HRF) or when targeting regions where cardiac pulsation plays an important role (42) not only in terms of signal intensity variation, but also in terms of motion]. Finally, at lower field strength, 3D RE-EPI is expected to perform better due to the reduced magnetic susceptibility effects and due to the longer $\mathrm{T}_{2}{ }^{*}$, allowing acquisition of a larger number of $\mathrm{k}$ space planes per segment.

\section{CONCLUSIONS}

We conclude that 3D RE-EPI, a novel acquisition scheme for 3D EPI that acquires two k-space planes per RF excitation, yields increased temporal resolution and better physiological noise characterization compared with 3D EPI while producing images with acceptable quality for fMRI studies. 3D RE-EPI (using a 32-channel coil, combined with conventional parallel imaging acceleration methods) allowed up to an eight-fold reduction of the number of excitations needed to obtain full brain coverage. This reduction in the number of excitations translated in acquisition acceleration in fMRI studies and improved tSNR per unit of time, which ultimately leads to an increase in the number of resting state networks being detected in $7 \mathrm{~T}$ resting state fMRI data.

\section{REFERENCES}

1. Mansfield P. Multi-planar image formation using NMR spin echoes. Journal of Physics C: Solid State Physics 1977;10:L55-L58.

2. Kwong KK, Belliveau JW, Chesler DA, et al. Dynamic magnetic resonance imaging of human brain activity during primary sensory stimulation. Proc Natl Acad Sci U S A 1992;89:5675-5679.

3. Ogawa S, Tank DW, Menon R, Ellermann JM, Kim SG, Merkle H, Ugurbil K. Intrinsic signal changes accompanying sensory stimulation: functional brain mapping with magnetic resonance imaging. Proc Natl Acad Sci U S A 1992;89:5951-5955.

4. Song AW, Wong EC, Hyde JS. Echo-volume imaging. Magn Reson Med 1994;32:668-671.

5. Mansfield P, Coxon R, Hykin J. Echo-volumar imaging (EVI) of the brain at $3.0 \mathrm{~T}$ : first normal volunteer and functional imaging results. J Comput Assist Tomogr 1995;19:847-852.

6. van der Zwaag W, Francis S, Bowtell R. Improved echo volumar imaging (EVI) for functional MRI. Magn Reson Med 2006;56:13201327.

7. Posse S, Ackley E, Mutihac R, Rick J, Shane M, Murray-Krezan C, Zaitsev M, Speck O. Enhancement of temporal resolution and BOLD sensitivity in real-time fMRI using multi-slab echo-volumar imaging. NeuroImage 2012;61:115-130.

8. Zahneisen B, Hugger T, Lee KJ, LeVan P, Reisert M, Lee HL, Asslander J, Zaitsev M, Hennig J. Single shot concentric shells trajectories for ultra fast fMRI. Magn Reson Med 2012;68:484-494.

9. Zahneisen B, Grotz T, Lee KJ, Ohlendorf S, Reisert M, Zaitsev M, Hennig J. Three-dimensional MR-encephalography: fast volumetric 
brain imaging using rosette trajectories. Magn Reson Med 2011;65: 1260-1268.

10. Abduljalil AM, Aletras AH, Robitaille PM. 3D echo planar imaging: application to the human head. Magn Reson Med 1995;34:144-148.

11. Muftuler LT, Nalcioglu O. Improvement of temporal resolution in fMRI using slice phase encode reordered 3D EPI. Magn Reson Med 2000;44:485-490.

12. Poser BA, Koopmans PJ, Witzel T, Wald LL, Barth M. Three dimensional echo-planar imaging at 7 Tesla. NeuroImage 2010;51:261-266.

13. Lai S, Glover GH. Three-dimensional spiral fMRI technique: a comparison with 2D spiral acquisition. Magn Reson Med 1998;39:68-78.

14. Hu Y, Glover GH. Three-dimensional spiral technique for highresolution functional MRI. Magn Reson Med 2007;58:947-951.

15. van der Zwaag W, Marques JP, Kober T, Glover G, Gruetter R, Krueger G. Temporal SNR characteristics in segmented 3D-EPI at 7T. Magn Reson Med 2012;67:344-352.

16. Glover GH, Li TQ, Ress D. Image-based method for retrospective correction of physiological motion effects in fMRI: RETROICOR. Magn Reson Med 2000;44:162-167.

17. Bianciardi M, Fukunaga M, van Gelderen P, Horovitz SG, de Zwart JA, Shmueli K, Duyn JH. Sources of functional magnetic resonance imaging signal fluctuations in the human brain at rest: a $7 \mathrm{~T}$ study. Magnetic resonance imaging 2009;27:1019-1029.

18. Jorge J, Figueiredo P, van der Zwaag W, Marques JP. Signal fluctuations in fMRI data acquired with 2D-EPI and 3D-EPI at 7 Tesla. Magn Reson Imaging 2013;31:212-220.

19. Larkman DJ, Hajnal JV, Herlihy AH, Coutts GA, Young IR, Ehnholm G. Use of multicoil arrays for separation of signal from multiple slices simultaneously excited. J Magn Reson Imaging 2001;13:313-317.

20. Setsompop K, Gagoski BA, Polimeni JR, Witzel T, Wedeen VJ, Wald LL. Blipped-controlled aliasing in parallel imaging for simultaneous multislice echo planar imaging with reduced g-factor penalty. Magn Reson Med 2012;67:1210-1224.

21. Nunes RG, Hanjal JV, Golay X, Larkman DJ. Simultaneous slice excitation and reconstruction for single shot EPI. In Proceedings of the 14th Annual Meeting of ISMRM. Seattle, Washington, USA, 2006. p. 293.

22. Moeller S, Yacoub E, Olman CA, Auerbach E, Strupp J, Harel N, Ugurbil K. Multiband multislice GE-EPI at 7 Tesla, with 16 -fold acceleration using partial parallel imaging with application to high spatial and temporal whole-brain fMRI. Magn Reson Med 2010;63: 1144-1153.

23. Feinberg DA, Moeller S, Smith SM, Auerbach E, Ramanna S, Gunther M, Glasser MF, Miller KL, Ugurbil K, Yacoub E. Multiplexed echo planar imaging for sub-second whole brain FMRI and fast diffusion imaging. PloS One 2010;5:e15710.

24. Feinberg DA, Reese TG, Wedeen VJ. Simultaneous echo refocusing in EPI. Magn Reson Med 2002;48:1-5.

25. Marques JP, Kober T, Krueger G, van der Zwaag W, Van de Moortele PF, Gruetter R. MP2RAGE, a self bias-field corrected sequence for improved segmentation and T1-mapping at high field. NeuroImage 2010;49:1271-1281.

26. Eggenschwiler F, Kober T, Magill AW, Gruetter R, Marques JP. SA2RAGE: a new sequence for fast B1+-mapping. Magn Reson Med 2012; 67:1609-1619.
27. Beckmann CF, DeLuca M, Devlin JT, Smith SM. Investigations into resting-state connectivity using independent component analysis. Philos Trans R Soc Lond B Biol Sci 2005;360:1001-1013.

28. Biswal B, Yetkin FZ, Haughton VM, Hyde JS. Functional connectivity in the motor cortex of resting human brain using echo-planar MRI. Magn Reson Med 1995;34:537-541.

29. Koopmans PJ, Boyacioglu R, Barth M, Norris DG. Whole brain, high resolution spin-echo resting state fMRI using PINS multiplexing at 7 T. NeuroImage 2012;62:1939-1946.

30. De Martino F, Esposito F, van de Moortele PF, Harel N, Formisano E, Goebel R, Ugurbil K, Yacoub E. Whole brain high-resolution functional imaging at ultra high magnetic fields: an application to the analysis of resting state networks. NeuroImage 2011;57:1031-1044.

31. Veer IM, Beckmann CF, van Tol MJ, Ferrarini L, Milles J, Veltman DJ, Aleman A, van Buchem MA, van der Wee NJ, Rombouts SA. Whole brain resting-state analysis reveals decreased functional connectivity in major depression. Front Syst Neurosci 2010;4.

32. Shumskaya E, Andriessen TM, Norris DG, Vos PE. Abnormal wholebrain functional networks in homogeneous acute mild traumatic brain injury. Neurology 2012;79:175-182.

33. Peters AM, Brookes MJ, Hoogenraad FG, Gowland PA, Francis ST, Morris PG, Bowtell R. T2* measurements in human brain at 1.5, 3 and 7 T. Magn Reson Imaging 2007;25:748-753.

34. Van de Moortele PF, Pfeuffer J, Glover GH, Ugurbil K, Hu X. Respiration-induced B0 fluctuations and their spatial distribution in the human brain at 7 Tesla. Magn Reson Med 2002;47:888-895.

35. van der Zwaag W, Marques JP, Hergt M, Gruetter R. Investigation of high-resolution functional magnetic resonance imaging by means of surface and array radiofrequency coils at $7 \mathrm{~T}$. Magn Reson Imaging 2009;27:1011-1018.

36. Jezzard P, Balaban RS. Correction for geometric distortion in echo planar images from B0 field variations. Magn Reson Med 1995;34:6573.

37. Zaitsev M, Hennig J, Speck O. Point spread function mapping with parallel imaging techniques and high acceleration factors: fast, robust, and flexible method for echo-planar imaging distortion correction. Magn Reson Med 2004;52:1156-1166.

38. Zeng H, Constable RT. Image distortion correction in EPI: comparison of field mapping with point spread function mapping. Magn Reson Med 2002;48:137-146.

39. Norris DG, Koopmans PJ, Boyacioglu R, Barth M. Power independent of number of slices (PINS) radiofrequency pulses for low-power simultaneous multislice excitation. Magn Reson Med 2011;66:12341240.

40. Bianciardi M, van Gelderen P, Duyn JH, Fukunaga M, de Zwart JA Making the most of fMRI at $7 \mathrm{~T}$ by suppressing spontaneous signal fluctuations. NeuroImage 2009;44:448-454.

41. Afacan O, Hoge WS, Janoos F, Brooks DH, Morocz IA. Rapid fullbrain fMRI with an accelerated multi shot 3D EPI sequence using both UNFOLD and GRAPPA. Magn Reson Med 2012;67:1266-1274.

42. Guimaraes AR, Melcher JR, Talavage TM, Baker JR, Ledden P, Rosen BR, Kiang NY, Fullerton BC, Weisskoff RM. Imaging subcortical auditory activity in humans. Hum Brain Map 1998;6:33-41. 